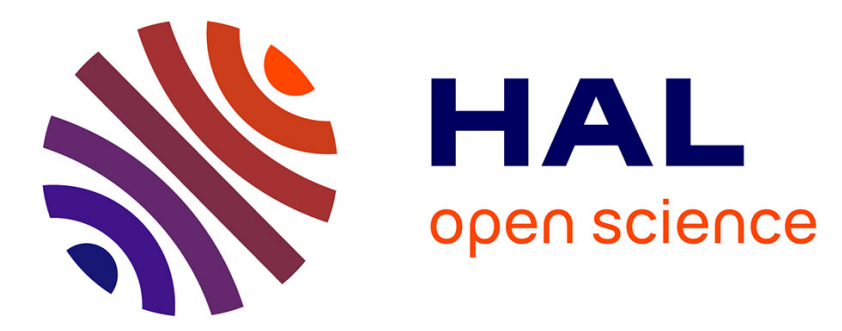

\title{
Recent Research and Development at Ircam
}

Hugues Vinet

\section{To cite this version:}

Hugues Vinet. Recent Research and Development at Ircam. Computer Music Journal, 1999, 23 (3), pp.9-17. hal-01250787

\section{HAL Id: hal-01250787 \\ https://hal.science/hal-01250787}

Submitted on 8 Jan 2016

HAL is a multi-disciplinary open access archive for the deposit and dissemination of scientific research documents, whether they are published or not. The documents may come from teaching and research institutions in France or abroad, or from public or private research centers.
L'archive ouverte pluridisciplinaire HAL, est destinée au dépôt et à la diffusion de documents scientifiques de niveau recherche, publiés ou non, émanant des établissements d'enseignement et de recherche français ou étrangers, des laboratoires publics ou privés. 
Recent Research and Development at IRCAM

Hugues Vinet

Institut de Recherche et Coordination Acoustique/Musique (IRCAM )

1 place Igor Stravinsky

F-75004 Paris

France

vinet@ircam.fr

Almost thirty years have elapsed since President Georges Pompidou proposed to Pierre Boulez the idea of creating a musical research institute that would be associated with the future Center for Contemporary Art in Paris, which later became the Centre Georges Pompidou. Since then, IRCAM developed its activities, while always remaining true to the goals defined at the outset: to be a place where composers, scientists, and engineers participate together in the renewal of contemporary music, while developing new knowledge and technologies related to musical composition, performance and listening. In 1991, Laurent Bayle succeeded Pierre Boulez as the head of IRCAM. One of his main concerns has been, together with setting up an efficient organization, to counter-balance a trend towards isolationism (which could have easily resulted from the specificity of its artistic and scientific content) by affirming IRCAM's role in society and by developing links to various types of public communities and fields of activity. This has been accomplished through greater interaction between IRCAM's two main poles of activity: musical production and Research and Development. The Education Department was set up under the direction of Jean-Baptiste Barrière and later MarieHélène Serra; it currently proposes various types of courses, including a one-year 
composition course, two nationally recognized graduate courses, a summer academy, week-end training sessions on IRCAM software, workshops for children, and so forth. The IRCAM Forum, part of the Marketing Department headed by Vincent Puig, and managed by Andrew Gerzso, was created in 1993 as a user group giving access to IRCAM software and various related services on a yearly subscription basis. The multimedia library, headed by Michael Fingerhut and officially opened in June 1996, gives access to a large set of documents centered around contemporary music: books, scores, digitized texts, photos, concert recordings, videos, CD-ROMs, and so forth. This trend towards eclecticism at IRCAM has been illustrated by the evolution of musical projects as a result of Risto Nieminen's, and then Eric de Visscher's, artistic direction. They have both been involved in developing exchanges with other artistic forms such as cinema, dance, opera and multimedia, while continuing to promote a culture of live musical performance that combines instruments and electronic parts as opposed to pure tape music.

This article focuses on the Research and Development activities at IRCAM, and aims at giving an overview of all of its projects presenting the general framework in that they operate. These activities take place in IRCAM's Research and Development Department, that has been in the recent past successively directed by Jean-François Allouis (1987-1991), Jean-Pascal Jullien (1991-1994), and myself since 1994.

\section{General Context of Research and Development Activities at IRCAM}

One main feature of IRCAM 's Research and Development activities is the combining of scientific research related to music with technological developments, mainly software-oriented, that aim at providing composers with new creative tools. 
All research fields, including acoustics, psycho-acoustics, digital signal processing, and various kinds of computer science, contribute to our developing knowledge and models related to various aspects of music production, even if their scope is broader. These models are then integrated into software tools with dedicated graphical interfaces that are mainly delivered as open environments and languages, so that they can be configured and adapted according to the various compositional needs and aesthetics. Modularity of the design is also a central concern, since it enables, together with better software maintenance, to integrate new models as soon as they are available from research. Since 1993, the development of the IRCAM Forum has had dramatic consequences on our software development, which had to evolve from personalized handwork to a collective production activity, and that currently delivers, with two releases a year, half a dozen different compositional environments to a community of nearly one thousand users. The Apple Macintosh has been the main target of software development in the past decade, although high-end workstations, like the IRCAM Signal Processing Workstation (ISPW) or more recently workstations from Silicon Graphics Inc., have been used when higher performance was required. We are also taking into account the PC world more and more, both with Windows and Linux operating systems.

In the past, there has been much emphasis on specific development projects, such as the ISPW, whereas fewer less means were allocated to other ones. The trend at IRCAM over the last years has been, on the contrary, to find equilibrium between various projects. We assume that there cannot be a single approach for renewing musical expression, and that greater richness can be globally achieved by developing and supporting several different approaches in parallel, while taking care of the way they communicate with each other for the sake of global coherence. 
Significant growth of research activities over the past years has been made possible by a systematic transfer of technologies and methodologies to the industry, coordinated by Vincent Puig in the Marketing Department, in various fields such as telecommunications, the automobile industry, audio production, multimedia entertainment, and online music distribution. Nevertheless, contributing to musical production remains the main raison d'être of all Research and Development activities at IRCAM, and different forms of communication and collaboration between researchers and musicians are defined and constantly adapted to various situations.

Each scientific field at IRCAM is covered by a specialized team that handles both research and development projects related to that field, so that the transfer from research to development is operated smoothly and continuously. The following paragraphs sum up the main features of recent work related to each of the fields.

\section{Acoustics}

As can be seen in IRCAM's acronym, research in acoustics has been an integral part of IRCAM's activity since the beginning. Current research is divided into instrumental acoustics and room acoustics.

\section{Instrumental Acoustics}

This research, headed by René Caussé, studies the behavior of musical instruments, including oscillation, resonance and radiation, from the viewpoint of acoustical modeling. Various families of instruments and excitations have been studied over the years, including brass instruments (Caussé, Kergomard, and Lurton 1984), clarinet (Maganza, Caussé, and Laloë 1986), bowed strings (Weinreich and Caussé 1991), recorder-like instruments (Verge et al. 1994) and piano, both in relation to the tuning of trichords, following G. Weinreich's work (Marandas, Caussé, and Gibiat 
1995) and to its radiation (Dérogis and Caussé 1995). Recent work also includes the study of non-linearity in the trombone at high intensities (Hirschberg et al. 1996).

This expertise has been applied to enhancing existing instruments and has led to the construction of a quarter-tone flute, a Wah-Wah mute for the horn and, more recently, a tunable clarinet embouchure controlled by a foot pedal, that enables the production of continuous pitch variations and micro-intervals without requiring special fingerings.

One main application of this research for composers is physical modeling synthesis. The team has developed an original approach based on modal synthesis, initiated by Jean-Marie Adrien (Adrien 1991), that led to the Mosaic environment (Morrison 1993), later renamed Modalys (Eckel, Iovino, and Caussé 1995; Iovino, Caussé, and Dudas 1997). As a modular environment that enables the user to build virtual instruments by assembling various objects coupled with various kinds of interactions, while enabling direct control of the sound components through the modal representation, Modalys can produce sounds than cannot be easily be obtained with other synthesis techniques. Current and future work includes the integration of new objects, interactions and modal data, the development of dedicated graphical interfaces (Polfreman 1997) and real time implementation using the jMax environment (Déchelle et al. 1999).

\section{Room Acoustics}

The Room Acoustics team was created more recently and has been successively headed by Jean-Pascal Jullien and Olivier Warusfel, the latter assisted by Jean-Marc Jot.

One of the team's research fields has been predictive acoustics. In relation to architectural design, the predictive computation of impulse responses is computed 
Vinet

from the data of the room's geometry and construction materials (Warusfel 1995a, Warusfel and Cruz 1995b). This expertise was applied to the design and the enhancement of concert halls, such as the Cité de la musique and Opéra Bastille in Paris.

Another topic of interest for composers that has received much attention over the recent years is related to the simulated spatialization of sound sources. This work is a result of combined studies of artificial reverberation (Jot 1992) and perception of the acoustical quality of concert halls; this latter research program identified a set of perceptively independent parameters which were then used for controlling simulation (Jullien 1995; Kahle and Jullien 1995). One appealing feature of this approach is that, unlike commercially available reverberators, it combines the simulation of source localization and room effect; that the spatialization effect can be specified independent of the reproduction system, so that, for example, the transcoding of multi-loudspeaker spatialization done in a concert into transaural or 5.1 surround formats for a CD or film production is computed automatically. This research led to the Spatialisateur, also known as the Spat (Jullien and Warusfel 1994; Jot 1997a), a modular, real-time spatialization environment based on the ISPW, which later became available in the Max/MSP and jMax environments. The Spat has been used by many composers, both for studio effects and in concert and has found many applications in other areas such as audio post-production, telecommunications, the automobile industry and virtual reality installations. Recent work has been dedicated to the implementation and enhancement of various reproduction formats, including intensity panning, binaural, transaural, Ambisonics, Dolby SR, source directivity and orientation, and development of connections between predictive acoustics and simulation tools (Jot, Larcher, and Warusfel 1995a; Jot 1997b). Future works will also be oriented towards radiation synthesis by means 
Vinet

of composite electro-acoustic sources (Warusfel, Dérogis and Caussé 1997), including the simulation of instrument radiation patterns and perceptive studies on source radiation.

\section{Music Perception and Cognition}

Research conducted by Steve McAdams aims at understanding the mental representation and the computational processes that intervene, at various levels, in music listening. The subject of the studies ranges from perceptive processes, closely related to physiology, to cognitive processes (McAdams 1989b, McAdams and Bigand 1993), whose features include, but are not exclusively limited to, culturedependent factors. The overall approach relies on the methodological framework of experimental psychology, statistical analysis, and computer-based modeling, which progressively build models of audition that account for phenomena of growing complexity by setting up experiments that rely on more and more complex stimuli.

Much work has been dedicated in the past to the study of auditory organization processes which are divided into sequential and simultaneous processes. Sequential organization refers to the way we group successive events into coherent flows (Beauvois and Meddis 1997), whereas simultaneous organization refers to the way our perception fuses acoustic components into a single source or segregates them into different ones (de Cheveigné et al. 1995; Marin and McAdams 1996; McAdams 1989a).

Studies on timbre perception from many different viewpoints make up a significant part of recent work done at IRCAM (McAdams and Cunibile 1992, McAdams 1999). Guest researcher Susan Winsberg's expertise in statistical analysis led to multidimensional scaling methods (Winsberg and de Soete 1993, 1997) that were adapted to the analysis of timbre spaces from experiments based on similarity 
Vinet

judgements (McAdams et al. 1995). These methods have been applied to the study of the acoustical quality of non-musical sources in various fields of activity such as the automobile industry and public transport (Susini, McAdams, and Winsberg 1999). The resulting quantitative similarity factors between different timbres were also used in the framework of the Studio Online project for computing a perceptive distance that was used for content-based retrieval in an audio sample database, related to earlier work (Kieslar et al. 1985; Misdariis et al. 1998). Timbre perception also has been studied in relation to the geometrical parameters of acoustic sources (Lakatos, McAdams, and Caussé 1997). Current and future research, together with the refinement of procedures developed for timbre perception, aim more and more at investigating the cognitive processes involved in the perception of musical structures (Pressnitzer et al. 1999).

\section{Sound Analysis and Synthesis}

Digital audio synthesis and processing and their related techniques have obviously represented a major field of interest for contemporary composers since the very beginning of computer music and they have constantly been refined over the years. The Analysis/Synthesis team at IRCAM headed by Xavier Rodet and assisted by Philippe Depalle, started by developing an expertise in voice synthesis including both synthesis algorithms (Galas and Rodet 1990) with the well-known Chant synthesizer (Rodet, Potard and Barrière 1984) and rule-based systems for synthesis control (Bennett and Rodet 1988; Cointe and Rodet 1984; Rodet and Delatre 1979).

This work has been extended to the design of various synthesis techniques that are based on a general analysis/synthesis framework including: (1) synthesis models, each of which is able to produce certain classes of sounds according to the values of a set of input parameters; (2) analysis models, whenever inversion is 
possible, that produce associated sets of parameters over time from real-world signals; (3) control systems, based on graphical or gestural interfaces and on sets of "performing" rules between synthesis parameters which ensure the consistency of the resulting synthesis. Sound processing associated with a given model can then be obtained through successive stages of analysis, modification of the resulting analysis parameters through adapted control, and re-synthesis using the new set of parameters.

Over the last years much emphasis has been put on signal models, such as the phase vocoder, and more particularly the sinusoidal model (Rodet 1997), for which efficient synthesis (Freed, Rodet and Depalle 1993; Rodet and Depalle 1992) and analysis (Rodet and Doval 1991; Depalle, Garcia, and Rodet 1993) procedures were developed. This expertise was applied to the creation of a castrato voice for the Farinelli film (Depalle, Garcia, and Rodet 1995a). It also led to the development of sound editing environments with graphical interfaces, such as the popular AudioSculpt program, which is based on the SVP phase vocoder, that provides timefrequency editing features controlled by graphic manipulations of the STFT sonogram. Diphone, a more recent development, is a synthesis control environment based on a technique developed for speech synthesis that enables the user to build musical phrases from the concatenation of elementary fragments, with possible control of various synthesis models such as additive synthesis and Chant (Rodet and Lefèvre 1997). Recent work related to signal models include refinements of analysis procedures and new approaches based on the extension of existing techniques such as wavelet analysis (Gribonval et al. 1996), pitch synchronous methods (Peeters and Rodet 1998) and statistical models (Dubnov and Rodet 1997). On the other hand, exploration of physical models has been done using the state variable formalism (Depalle and Tassart 1995b), with original implementations of fractional delays 
(Tassart and Depalle 1997), and from the viewpoint of non-linear dynamic systems, this latter work being extensively described by Rodet and Vergez in two (Rodet and Vergez 1999a, 1999b) articles in this issue.

Current and future work includes the enhancement and better availability of signal models in compositional environments, applications of signal models to automatic segmentation and the structuring of audio signals for automated search in audio databases, new interfaces and rule-based systems for synthesis control.

\section{Real-time Systems}

IRCAM has been a pioneer in designing real-time systems for live interaction between performers and computers, its main related achievements being the $4 \mathrm{X}$ processor (Di Guigno and Kott 1981, Di Guigno and Gerzso 1986; Favreau et al. 1986) and the IRCAM Musical Workstation (Lindemann et al. 1991), better known as the IRCAM Signal Processing Workstation (ISPW), based on NeXT computers with specialized DSP boards which were manufactured and distributed by Ariel Corporation. The Max software, formerly Patcher (Puckette 1988), from Miller Puckette, has been the main application used for programming and controlling realtime algorithms on the ISPW, while the Apple Macintosh version of Max was licensed to Opcode Systems Inc. and further developed by David Zicarelli with MSP, an ISPW compatible DSP library. This Macintosh version has been marketed worldwide with sales in the thousands and has since become a milestone in the design of interactive systems, not only in the field of music.

Dozens of composers have used the ISPW at IRCAM and elsewhere for pieces that combine real-time MIDI and digital audio processing. Standard synthesis and processing features have been proposed, as well as original developments such as the Spatialisateur library (Jot 1997a), Phase Aligned Formant synthesis objects 
(Puckette 1995), and various modules that perform pitch following and score recognition (Vercoe and Puckette 1985). The latest developments related to pitch following and score recognition were done for Philippe Manoury's "En Echo" for solo soprano and electronics (1993), and Pierre Boulez's "Anthème 2" for solo violin and electronics (1997).

Since the hardware associated with the ISPW has become obsolete, the main direction assigned to recent development, conducted by François Déchelle, has been to set up a software-only solution compatible with the ISPW patches, that can run on standard desktop computers and that can provide a solution guaranteeing continuity for existing pieces while building a foundation for future developments. This work has led to the jMax environment (Déchelle et al. 1998, 1999), which is totally compatible with the ISPW and represents a kind of optimal solution in terms of cross-platform capabilities, while being based on a software design that enables using modern operating system features such as symmetric multiprocessing and pre-emptive multithreading.

Current and future developments include new musical applications such as additive analysis / synthesis, physical modeling synthesis techniques such as Modalys (Iovino, Caussé and Dudas 1997) and non-linear systems (Rodet and Vergez 1999), and new score recognition algorithms and editors that combine other strategies, that are not limited to a note-by-note based pitch detection. A basic version of the environment for various platforms is available for free download on IRCAM 's ftp site.

\section{Computer Aided Composition}

The Musical Representation team at IRCAM, headed by Gérard Assayag, aims at developing Computer Aided Composition (CAC) tools, that is, software 
environments that enable the formalization and the computation of musical structures mainly in the context of instrumental composition. Despite earlier contributions in the field of formalized music by composers and researchers such as Iannis Xenakis (Xenakis 1992), Alfred Lehrdal (Lehrdal and Jackendoff 1985, 1986), Pierre Barbaud (Barbaud 1971), David Cope and André Riotte, the structuring of related research in the form of software tools available for composers is relatively recent at IRCAM, and has yielded two successive generations of CAC environments based on visual programming techniques: PatchWork, developed in collaboration with Mikael Laurson (Laurson 1996) and several composers, and OpenMusic (Assayag et al. 1997), IRCAM 's latest CAC environment. The history and the detailed features of these projects are presented in this issue in Agon et al. (1999), so the material treated in that text will not be covered here.

Since the time it became available, PatchWork has been adopted by numerous composers and it is hoped that OpenMusic, a new framework with a significantly increased expressiveness, will answer future needs. Two of the most innovative features are the extension of the visual programming aspects on the one hand, and the possibility of representing musical forms through a sophisticated sketch editor on the other hand. Future developments related to OpenMusic include the representation and manipulation of temporal structures, tighter integration of constraints, new libraries for synthesis control and automated assistance for orchestration.

\section{Studio Online}

As it can be seen over the last fifteen years, with the development of microcomputers and graphical interfaces, computer music applications have always closely followed and taken advantage of the evolution of the computer industry. 
Similarly, the Studio Online project, headed by Guillaume Ballet at IRCAM and funded in the context of the French Ministry of Industry's calls on Information Highways, explores new possibilities in the field of music production related to what could be a new generation of computing based on network connectivity and on a new distribution of programs and data between clients and servers.

The first kind of proposed services is an audio sample database that takes advantage of global access to all samples (as opposed to the usual CD-ROM distribution) by providing high level search features including access to multiphonics from their main salient pitches and content-based retrieval. The database content itself results from a two-year recording campaign under the artistic direction of Joshua Fineberg and then Fabien Lévy that produced samples of extensive contemporary playing modes of 16 instruments of the orchestra performed by international soloists, all recorded in 24 bits at $48 \mathrm{kHz}$ through six different microphones, yielding approximately 114,000 different sounds on 130 Gbytes.

The second kind of service is a library of processes that can be applied to database samples or to sounds uploaded by the user through the network. These processes do not fundamentally offer more features than those proposed in existing microcomputer applications, but their main advantage lies in that they are available through an integrated environment from any low cost, Java-compliant terminal with a Web browser while providing new possibilities for non-expert users. The project architecture (Woehrmann 1999), and in particular the use of the CORBA IIOP protocol coupled with a Java graphical interface has made Studio Online a real client/ server application on top of the Internet protocols. The main difficulty experienced in using Studio Online has been related to Internet's low bit rate, whereas access using IRCAM 's Intranet through a 10Mbits/s Ethernet LAN was quiet acceptable. Future developments may include domestic and educational 
applications using ADSL connections, real-time audio sample streaming, the extension of the existing database, the creation of new search procedures and new audio processes.

\section{Conclusion}

These descriptions illustrate the variety and the vitality of recent research and development at IRCAM that are due mainly to the personal involvement of many individuals, and to the significant and continuous support over years of the French Ministry of Culture.

\section{Acknowledgments}

Many thanks to Andrew Gerzso for proof reading this article.

\section{References}

Adrien, J.M. 1991. "The Missing Link: Modal Synthesis." In G. De Poli, A. Picalli, and C. Roads, eds. Representations of Musical Signals. Cambridge, Massachussets: MIT Press

Agon, C., G. Assayag, M. Laurson, C. Rueda, and O. Delerue. 1999. "Computer Assisted Composition at Ircam: PatchWork \& OpenMusic." Computer Music Journal 23(3): (this issue).

Assayag, G., C. Agon, J. Fineberg, and P. Hanappe. 1997."An Object Oriented Visual Environment For Musical Composition." Proceedings of the International Computer Music Conference. San Francisco: ICMA.

Barbaud, P. 1971. "La musique, discipline scientifique. Introduction élémentaire à l'étude des structures musicales." Paris, Dunod. 
Beauvois, M., and R. Meddis. 1997. "Time Decay of Auditory-Stream Biasing." Perception E Psychophysics 59, 81-86.

Bennett, G., and X. Rodet. 1988. "Synthesis of the Singing Voice." Proceedings of the Volume of the SDF Benchmark Series in the Area of Computer Music.

de Cheveigné, A., S. McAdams, J. Laroche, and M. Rosenberg. 1995. "Identification of Concurrent Harmonic and Inharmonic Vowels: A Test of the Theory of Harmonic Cancellation and Enhancement." Journal of the Acoustical Society of America 97, 3736-3748.

Caussé, R., J. Kergomard, and X. Lurton. 1984. "Input Impedance of Brass Musical Instruments: Comparison Between Experiments and Numerical Models." Journal of the Acoustical Society of America 75, 241-254.

Cointe, P., and X. Rodet. 1984. "FORMES: an Object \&Time Oriented System for Music Composition and Synthesis." In Conf. Rec. 1984 ACM Symp. on Lisp and Functional Programming. Austin, Texas, 5-8 Aug. 84.

Déchelle, F., et al. 1999. "jMax: an environment for real-time musical applications." Computer Music Journal 23(3): (this issue)

Déchelle, F., et al. 1998. "Latest Evolutions of the jMax Real-time Engine: Typing, Scoping, Threading, Compiling." Proceedings of the International Computer Music Conference. San Francisco: ICMA.

Depalle, P., G. Garcia, and X. Rodet. 1993. "Tracking of Partials for Additive Sound Synthesis using Hidden Markov Models." Proceedings of the International Conference Acoustics, Speech, and Signal Processing. Minneapolis. April 26-30, 1:225-228.

Depalle, P., G. Garcia, and X. Rodet. 1995a. “The Recreation of a Castrato Voice, Farinelli's Voice." Proceedings of the IEEE Acoustics, Speech, and Signal Processing 
Workshop on Applications of Signal Processing to Audio and Acoustics. New Paltz, New York: Mohonk Mountain House, Oct. 1995.

Depalle P., and S. Tassart. 1995b. "State Space Sound Synthesis and a State Space Synthesiser Builder." Proceedings of the of the International Computer Music Conference. San Francisco: ICMA.

Dérogis, P., and R. Caussé. 1995. "Computation and Modelisation of the Sound Radiation of an Upright Piano using Modal Formalism and Integral Equators." Proceedings of the 15th International Congress on Acoustics. Trondheim, 3:409-412.

Di Guigno, G., and A. Gerzso. 1986. "La station de travail musical 4X." IRCAM Technical Report. Paris: IRCAM.

Di Guigno, G., and J. Kott. 1981. "Présentation du système 4X, Processeur numérique de signal en temps réel." Ircam Report \#32, Paris: IRCAM available online at: http: / / mediatheque.ircam.fr/articles / textes /DiGiugno81a /

"Dubnov, S., and X. Rodet. 1997. "Statistical Modeling of Sound Aperiodicities." Proceedings of the International Computer Music Conference. San Francisco: ICMA. Eckel, G., F. Iovino, and R. Caussé. 1995. "Sound Synthesis by Physical Modelling with Modalys." Proceedings of the International Symposium on Musical Acoustics. Dourdan, France: pp. 478-482.

Favreau, E., et al. 1986. "Software Developments for the 4X Real-Time System." Proceedings of the International Computer Music Conference. San Francisco: ICMA, pp. 369-373

Freed, A., X. Rodet, and P. Depalle. 1993. "Synthesis and Control of Hundreds of Sinusoidal Partials on a Desktop Computer without Custom Hardware." Proceedings of the 1993 International Computer Music Conference. San Francisco: ICMA. 
Galas, T., X. Rodet. 1990. "A parametric Model of Speech Signals: Application to High Quality Speech Synthesis by Spectral and Prosodic Modifications." Proceedings of the ICSLP. Kobe, Japan, pp. 801-804.

Gribonval R., et al. 1996. “Analysis of Sound Signal with High Resolution Matching Pursuit." Proceedings of the IEEE Conference on Time-Frequency and Time-Scale Analysis (TFTS'96). Paris, France. June 1996.

Hirschberg, A., et al. 1996. "Shock Waves in Trombones." Journal of the Acoustical Society of America 99(3), 1754-1758.

Iovino, F., R. Caussé, and R. Dudas. 1997. "Recent work around Modalys and Modal Synthesis." Proceedings of the International Computer Music Conference, Thessaloniki, pp. 356-359.

Jot, J.M., 1992. "Etude et réalisation d'un spatialisateur de sons par modèles physique et perceptif." Ph.D. thesis TELECOM Paris 92 E 019, Paris: ENST

Jot, J.M., 1997a. "Efficient Models for Distance and Reverberation Rendering in Computer Music and Virtual Audio Reality." Proceedings of the International Computer Music Conference. San Francisco: ICMA.

Jot, J.M., L. Cerveau, and O. Warusfel, 1997b. "Analysis and Synthesis of Room Reverberation Based on a Statistical Time-Frequency Model." Proceedings of the 103rd AES Convention.

Jot, J.M., V. Larcher, and O. Warusfel. 1995a. "Digital Signal Processing Issues in the Context of Binaural and Transaural Stereophony." Proceedings of the 98th AES Convention. Paris.

Jullien, J.P. 1995. "Structured Model for the Representation and the Control of Room Aoustical Quality." Proceedings of the 15th International Congress on Acoustics. Trondheim. 
Jullien, J.P., and O. Warusfel. 1994. "Technologies et perception auditives de l'espace."

In Cahiers de l'Ircam $n^{\circ}$ 5. Paris: IRCAM. Available online at:

http: / / mediatheque/ articles / textes / Jullien94/

Kahle E., and J.P. Jullien. 1995. "Subjective Listening Tests in Concert Halls:

Methodology and Results." Proceedings of the 15th International Congress on Acoustics. Trondheim

Kieslar, D., et al. 1995. "Audio Analysis for Content-based Retrieval." Proceedings of the International Computer Music Conference. San Francisco: ICMA.

Lakatos, S., S. McAdams, and R. Caussé. 1997. "The Representation of Auditory Source Characteristics: Simple Geometric Form." Perception and Psychophysics 59(8): 1180-1190.

Laurson M. 1996. " PATCHWORK: A Visual Programming Language and some Musical Applications." Helsinki: Sibelius Academy.

Lerdahl, F., and R. Jackendoff. 1985. "A Generative Theory of Tonal Music."

Cambridge: MIT Press.

Lerdahl, F., and Y. Potard. 1986. "La composition assistée par ordinateur." IRCAM Report \#41, Paris, IRCAM, available online at: http: / / mediatheque.ircam.fr/articles / textes / Lerdahl86a/

Lindemann, E., et al. 1991. "The Architecture of the IRCAM Musical Workstation." Computer Music Journal 15(3):41-50.

Maganza, C., R. Caussé, and F. Laloë. 1986. "Bifurcations, Period Doublings and Chaos in Clarinetlike Systems." Europhys. letter, 1(6):295-302.

Marandas, E., R. Caussé, and V. Gibiat. 1995. "Tuning of Trichords in the Piano." Proceedings of the International Symposium on Musical Acoustics. Dourdan, July 2-6, pp. 312-316. 
McAdams, S. 1989a. "Segregation of Concurrent Sounds. I: Effects of Frequency

Modulation Coherence." Journal of the Acoustical Society of America. 86, 2148-2159.

McAdams, S. 1989b. "Psychological Constraints on Form-bearing Dimensions in Music." Contemporary Music Review 4:181-198.

McAdams, S. 1999. " Perspectives on the Contribution of Timbre to Musical

Structure." Computer Music Journal 23(3): (this issue)

McAdams, S., and E. Bigand (Eds.). 1993. Thinking in Sound: The Cognitive Psychology of Human Audition. Oxford: Clarendon Press.

McAdams, S., and J. C. Cunibile 1992. "Perception of Timbral Analogies." Philosophical Transactions of the Royal Society, London, series B 336:383-389.

McAdams, S., et al. 1995. "Perceptual Scaling of Synthesized Musical Timbres:

Common Dimensions, Specificities, and Latent Subject Classes." Psychological Research 58:177-192.

Marin, C., and S. McAdams 1996. "The Role of Frequency Modulation, Polyperiodicity, and Auditory Beats in the Recognition of Spectrally Embedded Complex Target Sounds." Journal of the Acoustical Society of America 100:1736-1753.

Misdariis, N., et al. 1998. "Validation of a multidimensional distance model for perceptual dissimilarities among musical timbres." Proceedings of the 16th International Congress on Acoustics. Seattle, Woodbury, NY: ASA.

Morrison, J., J.M. Adrien. 1993. " MOSAIC: A Framework for Modal Synthesis." Computer Music Journal 17(1):45-46.

Peeters, G., X. Rodet. 1998. "Sinusoidal Characterization in Terms of Sinusoidal and Non-Sinusoidal Components." DAFX98-Workshop, Barcelona-Spain.

Polfreman, R. 1997. "User-Interface Design for Software Based Sound Synthesis Systems.". Ph. D. Thesis, University of Hertfordshire, United Kingdom. 
Pressnitzer, D., et al. 1999. "Perception of Musical Tension for Nontonal Orchestral Timbres and its Relation to Psychoacoustic Roughness." Perception $\mathcal{E}$ Psychophysics (in press).

Puckette, M. 1988. "The Patcher." Proceedings of the International Computer Music Conference. San Francisco: ICMA, pp. 420-425.

Puckette, M. 1995. "Formant-Based Audio Synthesis Using Nonlinear Distorsion." Journal of the Audio Engineering Society, Vol 43, No 1/2, 1995 January / February

Rodet, X., and J.L. Delatre. 1979. "Time-domain Speech Synthesis by Rule using a flexible and fast signal management system." Proceedings of the IEEE International Conference on Acoustics, Speech, and Signal Processing. Washington, D.C., April 1979

Rodet, X., Y. Potard, and J.B. Barrière. 1984. "The CHANT Project: From Synthesis of the Singing Voice to Synthesis in General." Computer Music Journal. 8(3): 15-31

Rodet, X., and B. Doval. 1991. "Estimation of Fundamental Frequency of Musical Sound Signals." Proceedings of the IEEE International Conference on Acoustics, Speech, and Signal Processing. May 1991, Toronto.

Rodet, X., and P. Depalle. 1992. "Spectral Envelopes and Inverse FFT Synthesis." Proceedings of the 93rd AES Convention. San Francisco: AES, New York, October 1992.

Rodet, X. 1997. “Musical Sound Signals Analysis/Synthesis: Sinusoidal + Residual and Elementary Waveform Models." Applied Signal Processing. 1997. 4:131-141

Rodet, X., and A. Lefèvre. 1997. "The Diphone Program: New Features, New Synthesis Methods and Experience of Musical Use." Proceedings of the International Computer Music Conference. San Francisco: ICMA pp 418-421 
Vinet

Rodet, X., and C. Vergez. 1999. "Nonlinear Dynamics in Physical Models: Simple Feedback Loop Systems and Properties." Computer Music Journal 23(3): (this issue)

Rodet, X., and C. Vergez. 1999. " Nonlinear Dynamics in Physical Models: From Basic Models to True Musical Instrument Models. " Computer Music Journal 23(3): (this issue)

Tassart, S., and P. Depalle. 1997. "Analytical Approximations of Fractional Delays: Lagrange Interpolators and Allpass Filters." International Conference on Acoustics, Speech, and Signal Processing. April 21-24, 1997, Munich, Germany

Susini, P., S. McAdams, and S. Winsberg. 1999. "Perceptual Characterisation of Vehicle Noises." Acta Acustica (in press).

Vercoe, B., and M. Puckette. 1985. "Synthetic Rehearsal: Training the Synthetic Performer." Proceedings of the International Computer Music Conference. San Francisco: ICMA.

Verge, M.P., et al. 1994. "Jet Oscillations and Jet Drive in Recorder-like Instruments." Acta Acustica. 2, 403-419.

Warusfel, O. 1995a. "Predictive Acoustics Software and Computer Aided Optimization in Room Acoustics." Proceedings of the 15th International Congress on Acoustics. Trondheim.

Warusfel, O., and F. Cruz. 1995b. "Validation of a Computer Model Environment for Room Acoustics Prediction." Proceedings of the 15th International Congress on Acoustics. Trondheim.

Warusfel, O., P. Dérogis, and R. Caussé. 1997. "Radiation Synthesis with Digitally Controlled Loudspeakers." Preprint 4577 (K-5), 103rd AES Convention Weinreich, G., and R. Caussé. 1991. "Elementary Stability Considerations for BowedString Motion." Journal of the Acoustical Society of America 89, 887-895. 
Winsberg, S., and G. de Soete 1993. "A Latent Class Approach to Fitting the Weighted Euclidean Model, CLASCAL." Psychometrika 58: 315-330.

Winsberg, S., and G. de Soete. 1997. "Multidimensional Scaling with Constrained Dimensions: CONSCAL." British Journal of Mathematical and Statistical Psychology 50:55-72.

Winsberg, S., and G. de Soete 1993. " A Latent Class Approach to Fitting the Weighted Euclidean Model, CLASCAL." Psychometrika 58: 315-330.

Winsberg, S., and G. de Soete. 1997. "Multidimensional Scaling with Constrained Dimensions: CONSCAL." British Journal of Mathematical and Statistical Psychology 50: 55-72.

Wöhrmann, R. 1999. "Design and Architecture of Distributed Sound Processing and Database Systems for Web Based Computer Music Applications." Computer Music Journal 23(3): (this issue)

Xenakis, I. 1992. Formalized Music: Thought and Mathematics in Composition. New ed. Stuyvesant: Pendragon Press 